\title{
A NEW VECTOR QUASI-EQUILIBRIUM-LIKE PROBLEM
}

\author{
Byung-Soo LeE
}

\begin{abstract}
In this paper, we consider the existence of solutions to some generalized vector quasi-equilibrium-like problem under a $c$-diagonal quasi-convexity assumptions, but not monotone concepts. For an example, in the proof of Theorem 1, the $c$-diagonally quasi-convex concepts of a set-valued mapping was used but monotone condition was not used. Our problem is a new kind of equilibrium problems, which can be compared with those of Hou et al. [4].
\end{abstract}

\section{Introduction and preliminaries}

Equilibrium problems are important parts in nonlinear analysis connecting with variational inequality problems, optimization problems, complementarity problems, fixed point theorems and so on $[1,4,5,6,7,10]$.

In consideration the existences of solutions to equilibrium problems and its applications, the continuity, the monotonicity or the convexity of the core mappings are very meaningful.

In 2003, Ansari and Bazan [1] considered, so called, generalized vector quasiequilibrium problems for condensing set-valued mappings in Hausdorff topological vector spaces;

Find $\bar{x} \in K$ such that

$$
\bar{x} \in A(\bar{x}) ; \quad F(\bar{x}, y) \not \subset-\operatorname{int} C(x) \text { for all } y \in A(\bar{x}) .
$$

In 2003, Hou, Yu, and Chen [4] considered the following vector quasi-equilibrium problems for set-valued mappings under $c$-diagonal quasi-convexity assumptions;

Find $(\bar{x}, \bar{y}) \in X \times Y$ such that $\bar{x} \in S(\bar{x}), \bar{y} \in T(\bar{x})$ and

$$
f(\bar{x}, \bar{y}, u) \not \subset-\operatorname{int} C(\bar{x}) \text { for } u \in S(\bar{x}),
$$

where $E, W$ and $Z$ are Hausdorff topological vector spaces, $X$ and $Y$ are nonempty subsets of $E$ and $W$, respectively. And $S: X \rightarrow 2^{X}, T: X \rightarrow 2^{Y}$,

Received February 3, 2009; Revised May 14, 2009.

2000 Mathematics Subject Classification. 49J40.

Key words and phrases. vector quasi-equilibrium-like problem, $c$-diagonally quasi-convex, open lower sections, Browder fixed point theorem.

This research was supported by Kyungsung University Research Institute Grants in 2008. 
$f: X \times Y \times X \rightarrow 2^{Z}$ are set-valued mappings and $C: X \rightarrow 2^{Z}$ is a set-valued mapping with nonempty interior of $C(x)$, int $C(x)$ for all $x \in X$.

Let $L(E, Z)$ be the space of all continuous linear mappings from $E$ to $Z$, which is equipped with a $\sigma$-topology. Then $L(E, Z)$ is also a Hausdorff topological vector space [8].

Putting $Y$ to be a nonempty subset of $L(E, Z)$ instead of $W$ in (1.1), and adding a set-valued mapping $A: L(E, Z) \rightarrow 2^{L(E, Z)}$ and a single-valued mapping $\eta: X \times X \rightarrow E$, we obtain a new vector quasi-equilibrium-like problem (VQELP) for set-valued mappings as follows;

Find $(\bar{x}, \bar{y}) \in X \times Y$ such that $\bar{x} \in S(\bar{x}), \bar{y} \in T(\bar{x})$ and

$$
\langle w, \eta(u, \bar{x})\rangle+f(\bar{x}, \bar{y}, u) \not \subset-\operatorname{int} C(\bar{x}) \text { for } u \in S(\bar{x}) \text { and } w \in A(\bar{y}) .
$$

In this paper, we consider the existence of solutions to (VQELP) with the $c$-diagonal quasi-convexity, but not monotone concepts.

\section{Quasi-equilibrium-like problem}

The concept of the $c$-diagonal quasi-convexity was introduced by Hou et. al. [4]. The following lemmas are essential to prove our result.

Definition 1. Let $X$ be a convex subset of a topological vector space $E$ and $Z$ be a topological vector space. Let $C: X \rightarrow 2^{Z}$ and $f: X \times X \rightarrow 2^{Z}$ be set-valued mappings. $f$ is said to be $c$-diagonally quasi-convex in the second argument if for some $x_{i} \in M$,

$$
f\left(x, x_{i}\right) \nsubseteq-\operatorname{int} C(x),
$$

for $x \in \operatorname{coM}$, the convex hull of a finite subset $M=\left\{x_{i}: i=1,2, \ldots, n\right\}$ of $X$, i.e., $x=\sum_{i=1}^{n} \alpha_{i} x_{i}$ with $\alpha_{i} \geq 0(i=1, \ldots, n)$ and $\sum_{i=1}^{n} \alpha_{i}=1$.

A set-valued mapping $T: X \rightarrow 2^{Y}$ is said to have open lower sections if its fibers

$$
T^{-}(y)=\{x \in X: y \in T(x)\} \quad \text { is open in } X \text { for every } y \in Y \text {. }
$$

Lemma 1 ([9]). Let $X$ and $Y$ be topological spaces, and $S, T: X \rightarrow 2^{Y}$ be set-valued mappings with open lower sections. Then

(i) A set-valued mapping $F: X \rightarrow 2^{Y}$ defined by $F(x)=\operatorname{coS}(x)$, for each $x \in X$, has open lower sections.

(ii) A set-valued mapping $G: X \rightarrow 2^{Y}$ defined by $G(x)=S(x) \cap T(x)$, for each $x \in X$, has open lower sections.

Lemma $2([2])$. Let $X_{i}(i \in I)$ be a nonempty compact convex subset of a Hausdorff topological vector space $E_{i}(i \in I)$ and $E=\prod_{i \in I} E_{i}, X=\prod_{i \in I} X_{i}$, where $I$ is an index set. If set-valued mappings $T_{i}: X \rightarrow 2^{X_{i}}(i \in I)$ have convex set-values and $X=\bigcup_{x_{i} \in X_{i}}$ int $T_{i}^{-}\left(x_{i}\right)$, then there exists $\bar{x}\left(:=\left(\bar{x}_{i}\right)_{i \in I}\right) \in$ $X$ such that $\bar{x} \in \prod_{i \in I} T_{i}(\bar{x})$, i.e., $\bar{x}_{i} \in T_{i}(\bar{x})$, where $\bar{x}_{i}$ is a projection of $\bar{x}$ onto $X_{i}$. 
Browder fixed point theorem [3]. Let $X$ be a nonempty compact convex subset of a Hausdorff topological vector space $E$. Suppose that $F: X \rightarrow 2^{X}$ is a set-valued mapping with nonempty convex set-values and open lower sections, then $F$ has a fixed point.

Theorem 1. Let $E$ and $Z$ be Hausdorff topological vector spaces, and $X$ be a nonempty compact convex subset of $E$. Let $Y$ be a nonempty compact convex subset of a Hausdorff topological vector space $L(E, Z)$ equipped with a $\sigma$ topology. Let $A: L(E, Z) \rightarrow 2^{L(E, Z)}, f: X \times Y \times X \rightarrow 2^{Z}, S: X \rightarrow 2^{X}$ and $T: X \rightarrow 2^{Y}$ be set-valued mappings, $\eta: X \times X \rightarrow E$ be a single-valued mapping and $C: X \rightarrow 2^{Z}$ be a set-valued mapping with int $C(x) \neq \emptyset$ for $x \in X$.

Let the following conditions be satisfied;

(i) $S$ and $T$ have nonempty convex set-values and open lower sections,

(ii) for $y \in Y$ and $x \in \operatorname{co} M$, where $M=\left\{x_{i}: i=1,2, \ldots, n\right\}$ is any finite subset of $X$, a set-valued mapping $\ell: X \rightarrow 2^{Z}$ defined by, for each $u \in X$,

$$
\ell(u)=\langle w, \eta(u, x)\rangle+f(x, y, u) \quad \text { for some } w \in A(y)
$$

is c-diagonally quasi-convex, i.e.,

$$
\ell\left(x_{i}\right)=\left\langle w, \eta\left(x_{i}, x\right)\right\rangle+f\left(x, y, x_{i}\right) \nsubseteq-\operatorname{int} C(x) \quad \text { for some } w \in A(y),
$$

(iii) for $u \in X$, a set

$$
K=\{(x, y) \in X \times Y:\langle w, \eta(u, x)\rangle+f(x, y, u) \subseteq-\operatorname{int} C(x) \text { for } w \in A(y)\}
$$

is open.

Then there exists $(\bar{x}, \bar{y}) \in X \times Y$ such that $\bar{x} \in S(\bar{x})$ and $\bar{y} \in T(\bar{x})$ satisfying

$$
\langle w, \eta(u, \bar{x})\rangle+f(\bar{x}, \bar{y}, u) \nsubseteq-\operatorname{int} C(\bar{x})
$$

for $u \in S(\bar{x})$ and $w \in A(\bar{y})$.

Proof. Define a set-valued mapping $P: X \times Y \rightarrow 2^{X}$ by, for $(x, y) \in X \times Y$,

$$
P(x, y)=\{u \in X:\langle w, \eta(u, x)\rangle+f(x, y, u) \subseteq-\operatorname{int} C(x) \text { for } w \in A(y)\},
$$

then

(a) for all $(x, y) \in X \times Y, x \notin \operatorname{coP}(x, y)$.

If not, there exists a point $(\bar{x}, \bar{y}) \in X \times Y$ such that $\bar{x} \in \operatorname{coP}(\bar{x}, \bar{y})$. Hence there exists a finite subset $M=\left\{x_{i}: i=1,2, \ldots, n\right\} \subset P(\bar{x}, \bar{y})$ for $\bar{x} \in \operatorname{coM}$ such that

$$
\left\langle w, \eta\left(x_{i}, \bar{x}\right)\right\rangle+f\left(\bar{x}, \bar{y}, x_{i}\right) \subseteq-\operatorname{int} C(\bar{x}) \text { for } w \in A(\bar{y}),
$$

which contradicts condition (ii).

(b) $P$ has open lower sections.

In fact, for each $u \in X$,

$P^{-}(u)=\{(x, y) \in X \times Y:\langle w, \eta(u, x)\rangle+f(x, y, u) \subseteq-\operatorname{int} C(x)$ for $w \in A(y)\}$ is open by condition (iii). 
Define a set-valued mapping $G: X \times Y \rightarrow 2^{X}$ by, for $(x, y) \in X \times Y$,

$$
G(x, y)=\operatorname{coP}(x, y) \cap S(x),
$$

then

(c) $G$ has open lower sections.

In fact, by condition (i) $S$ has open lower sections and, by (b) and Lemma 1(i) $c P$ has open lower sections, hence by Lemma 1(ii) $G$ has open lower sections.

Put $W=\{(x, y) \in X \times Y: G(x, y) \neq \emptyset\}$.

(d) If $W=\emptyset$, then by the definition of $G(x, y)$,

$$
P(x, y) \cap S(x)=\emptyset \quad \text { for }(x, y) \in X \times Y .
$$

On the other hand, since $X$ is a compact convex subset of $E$ and $S$ is a convex set-valued mapping, by Browder fixed point theorem from condition (i) there exists $\bar{x} \in S(\bar{x})$. Since $T(\bar{x}) \neq \emptyset$, by taking $\bar{y} \in T(\bar{x})$ we have

$$
P(\bar{x}, \bar{y}) \cap S(\bar{x})=\emptyset .
$$

Hence for $u \in S(\bar{x}), u \notin P(\bar{x}, \bar{y})$, i.e., for $u \in S(\bar{x})$,

$$
\langle w, \eta(u, \bar{x})\rangle+f(\bar{x}, \bar{y}, u) \nsubseteq-\operatorname{int} C(\bar{x}) \quad \text { for } w \in A(\bar{y}) .
$$

(e) If $W \neq \emptyset$, then we define a set-valued mapping $M: X \times Y \rightarrow 2^{X}$ by, for each $(x, y) \in X \times Y$

$$
M(x, y)= \begin{cases}G(x, y), & (x, y) \in W, \\ S(x), & (x, y) \in X \times Y \backslash W .\end{cases}
$$

Then for each $(x, y) \in X \times Y, M(x, y)$ is convex, and for $x \in X, M^{-}(x)=$ $G^{-}(x) \cup\left(S^{-}(x) \times Y\right)$ is open.

Define a set-valued mapping

$$
H: X \times Y \rightarrow 2^{X \times Y}
$$

by, for each $(x, y) \in X \times Y, H(x, y)=(M(x, y), T(x))$, where $H_{1}(x, y)=$ $(M(x, y))$ and $H_{2}(x, y)=T(x)$, then by the assumption on $T$ in condition (i) and the properties of $M(x, y), H$ satisfies conditions of Lemma 2, i.e.,

$$
\begin{aligned}
X \times Y & =\bigcup_{\substack{x \in X \\
y \in Y}} H^{-}(x, y) \\
& =\bigcup_{\substack{x \in X \\
y \in Y}}\left(M^{-}(x) \cup T^{-}(y)\right) \\
& =\left(\bigcup_{x \in X} M^{-}(x)\right) \bigcup\left(\bigcup_{y \in Y} T^{-}(y)\right)
\end{aligned}
$$




$$
\begin{aligned}
& =\bigcup_{x \in X}\left(G^{-}(x) \cup\left(S^{-}(x) \times Y\right)\right) \bigcup\left(\bigcup_{y \in Y} T^{-}(y)\right) \\
& =\left(\bigcup_{x \in X} \operatorname{int} H_{1}^{-}(x)\right) \bigcup\left(\bigcup_{y \in Y} \operatorname{int} H_{2}^{-}(y)\right) .
\end{aligned}
$$

Hence, there exists $(\bar{x}, \bar{y}) \in X \times Y$ such that $(\bar{x}, \bar{y}) \in H(\bar{x}, \bar{y})$. Thus we obtain $(\bar{x}, \bar{y}) \notin W$.

In fact, if $(\bar{x}, \bar{y}) \in W$, then $G(\bar{x}, \bar{y}) \neq \emptyset$. Hence $\bar{x} \in \operatorname{coP}(\bar{x}, \bar{y}) \cap S(\bar{x})$, so that $\bar{x} \in \operatorname{coP}(\bar{x}, \bar{y})$, which is a contradiction to (a). Since $(\bar{x}, \bar{y}) \in X \times Y \backslash W,(\bar{x}, \bar{y}) \in$ $H(\bar{x}, \bar{y})=(M(\bar{x}, \bar{y}), T(\bar{x}))=(S(\bar{x}), T(\bar{x}))$, and $G(\bar{x}, \bar{y})=\emptyset$. Consequently, $\bar{x} \in S(\bar{x}), \bar{y} \in T(\bar{x}), \operatorname{coP}(\bar{x}, \bar{y}) \cap S(\bar{x})=\emptyset$. Thus

$$
P(\bar{x}, \bar{y}) \cap S(\bar{x})=\emptyset .
$$

Hence for $u \in S(\bar{x})$,

$$
\langle w, \eta(u, \bar{x})+f(\bar{x}, \bar{y}, u)\rangle \not-\operatorname{int} C(\bar{x}) \text { for } w \in A(\bar{y}) .
$$

Remark. Problem (1.2) can be compared with Hou et al.'s problem (1.1) in the point of view that the term $\langle w, \eta(u, \bar{x})\rangle$ is added and $Y$ is a subset of a particular Hausdorff topological vector space $L(E, Z)$ instead of an arbitrary Hausdorff topological vector space $W$.

\section{References}

[1] Q. H. Ansari and F. F. Bazan, Generalized vector quasi-equilibrium problems with applications, J. Math. Anal. Appl. 277 (2003), 246-256.

[2] Q. H. Ansari and J. C. Yao, A fixed point theorem and its applications to the system of variational inequalities, Bull. Aust. Math. Soc. 59 (1990), 433-442.

[3] F. E. Browder, The fixed point theory of multivalued mappings in topological vector spaces, Math. Annal. 177 (1968), 283-301.

[4] S. H. Hou, H. Yu, and G. Y. Chen, On vector quasi-equilibrium problems with set-valued maps, J. Optim. Th. Appl. 119 (2003), 485-498.

[5] L.-J. Lin, S. Park, and Z.-T. Yu, Remarks on fixed points, maximal elements, and equilibria of general games, J. Math. Anal. Appl. 233 (1999), 581-596.

[6] Q. M. Liu, L. Fan, and G. Wang, Generalized vector quasi-equilibrium problems with set-valued mappings, Appl. Math. Lett. 21 (2008), 946-950.

[7] M. A. Noor, Mixed quasi-equilibrium-like problems, J. Appl. Math. Stoch. Anal. (2006), Art. ID 70930, 1-8.

[8] H. H. Schaefer, Topological Vector Spaces, Graduate Texts in Mathematics, Vol. 3, Springer, New York, 1971.

[9] X. Q. Yang, Generalized convex functions and vector variational inequalities, J. Optim. Th. Appl. 79 (1993), 563-580.

[10] G. X.-Z. Yuan, G. Isac, K.-K. Tan, and J. Yu, The study of minimax inequalities, abstract economics and applications to variational inequalities and Nash equilibria, Acta Appl. Math. 54 (1998), 135-166. 
Basic Science Research Center

Kyungsung University

Busan 608-736, KoreA

E-mail address: bslee@ks.ac.kr 